\title{
Disappearance and Reappearance of Dust Particles in Cogenerated Dusty Plasma
}

\author{
Malay Mondal, ${ }^{1}$ Sanjib Sarkar, ${ }^{2}$ S. Mukherjee ${ }^{3} \&$ M. Bose, ${ }^{1 *}$ \\ ${ }^{I}$ Department of Physics, Jadavpur University, Kolkata 700032, India ${ }^{2}$ Institute of Plasma Physics, Chinese \\ Academy of Sciences, PO Box 1126, Hefei, Anhui 230031, People's Republic of China ${ }^{3}$ FCIPT, Institute for \\ Plasma Research, Gandhinagar 382428, India
}

\begin{abstract}
In this unique experiment, where dust is produced in a sub-atmospheric pressure plasma formed between two parallel plate electrodes using a bipolar pulsed dc power supply as the source of power for plasma production, dust disappearance for few seconds is experimented. The plasma forming gas is a mixture of argon and acetylene. The plasma phase chemical reactions help in the formation of sub-micron dust particles that grow in size between two parallel electrodes. Using another single negative pulse, placed in between two electrodes, applied to another electrode, the dust particles can be disappeared for significant duration from the dusty plasma zone. The applied single pulse voltage is varied between 50-250 V. On the application of the pulse, in its initial phase, the dusty plasma gets disturbed and for some duration ( few seconds) there is no light scattering observed, indicating that the dust is disappeared from the region between the electrodes. Later the plasma formation again reappears and similar structured dusty cloud is formed. The results are important as it gives an idea how to displace some unwanted dusts from our required region to another region.
\end{abstract}

Keywords: Dusty plasma, cogenerated, disappearance and reappearance

\section{INTRODUCTION:}

Dust particles, grows in plasma processing reactors by reactive gases or by electrode sputtering severely affects the quality of the processed products. Also in fusion devices they limit the efficiency of fusion reaction. Therefore, disappearance of dust particles for any typical condition seems to be very much important in present day plasma experiments. Various methods have been deployed by several workers for removal of unwanted dust from plasma, like, fine particle removal by a negatively-charged fine particle collector in silane plasma [1], dust removal by traveling plasma sheath distortion by modulating the voltage signal on striped electrode [2], removal of floating dust using pulsed plasma jet [3], electromagnetic radiation pressure from high power laser beam [4]. Production of dust can also be suppressed by using hydrogen gas in reactive plasma have also been reported [5]. In spite of advantages, proposed methods also have limitation like there exists an optimal laser frequency for which the laser power and energy has minimum value for plasma disappearance. As the method is effective in small region, more source energy is required to cover the wider volume.

The application of negative electric pulse to dust cloud was employed for studying the properties of dust acoustic solitary wave (DASW). Here, we have shown that, by varying pulse height and width (also the discharge parameters), a negative electric pulse is also effective for disappearance of dust particles from a particular region.

\section{EXPERIMENTS AND RESULTS:}

Cogenerated plasma (a continuously generated mixture of carbon particles and acetylene in argon plasma) with dust particles [6,7]are produced in our system. Two parallel plate electrodes of dimensions $100 \times$ $150 \mathrm{~mm}^{2}$ at $5 \mathrm{~cm}$ apart are placed inside the chamber. Graphite plate was mounted on upper electrode. We apply bipolar dc power supply (output voltage - 400v (max), output current - 0-1A (max), frequency - 50kHz, duty cycle $-25 \%$ ) to graphite (upper) electrode for sputtering, while the lower electrode was grounded. A schematic diagram of our experimental set up is shown in Fig. 1. The whole system was evacuated to base pressure of 10 ${ }^{5}$ mbar by means of a combination of rotary and diffusion pumps. The discharge was initiated by 500 peak-topeak voltage with 0.05 mbar of argon gas. Acetylene gas is then introduced, which acts as a monomer and initiate polymerization through collision with electrons leading to strong and rapid powder formation. As we gradually increase the peak-to-peak voltage, carbon dusts appear due to sputtering from graphite and dissociation due to acetylene, which then levitated between two electrodes. We then illuminated dust particles by a combination of He-Ne laser $(20 \mathrm{~mW}$, wavelength $630 \mathrm{~nm})$ and a cylindrical lens. We use CCD camera (BASLER-make) to observe scattered light from the dust particles at a direction angular to the laser sheet at a rate of 60 frames per second (fps). The dust particles are in dynamic phase, i.e. they are growing in plasma through various stages like nucleation, agglomeration and coagulation. Dust particles were levitated between 
electrodes in layer arrangement and acquire negative charge $\left(10^{3} \mathrm{e}-10^{4} \mathrm{e}\right)$ in laboratory plasma. These particles are massive compared to electrons and ions with a typical frequency of several tens of $\mathrm{Hz}$.

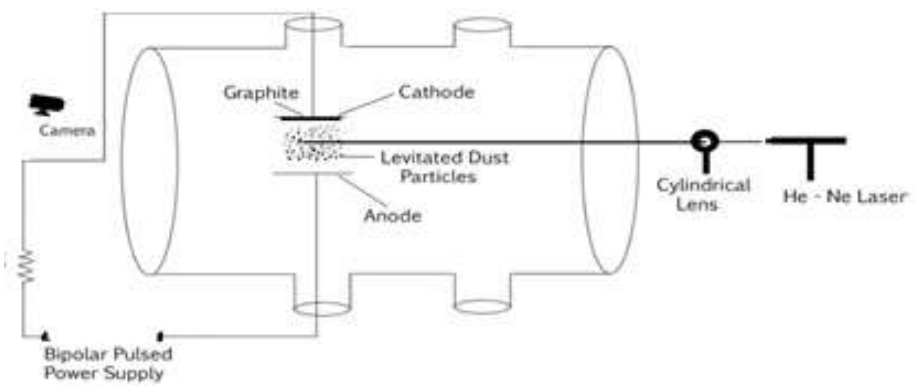

FIG.1. Schematic diagram of our experimental set-up.

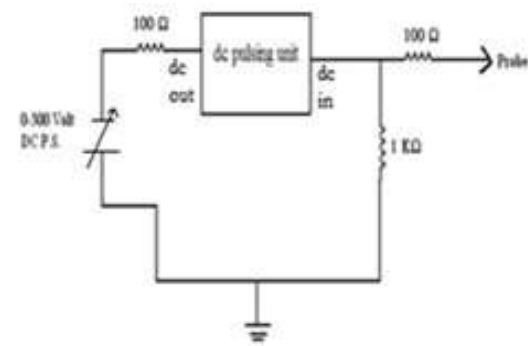

FIG.2. Pulse forming circuit.

A cylindrical electrode (length $65 \mathrm{~mm}$ and radius $1 \mathrm{~mm}$ ) is placed inside the chamber behind the dust cloud for introducing the pulse. Schematic diagram of pulsing circuit is given in Fig.2 and typical voltage pulse shown in Fig.3. Single negative pulse is given to the dust cloud through the electrode. Therefore, by varying pulse width $(125 \mathrm{~ms}-5 \mathrm{~s})$ with sufficient pulse voltages $(50 \mathrm{v}-250 \mathrm{v})$, dust particles can be knocked out of plasma trap.

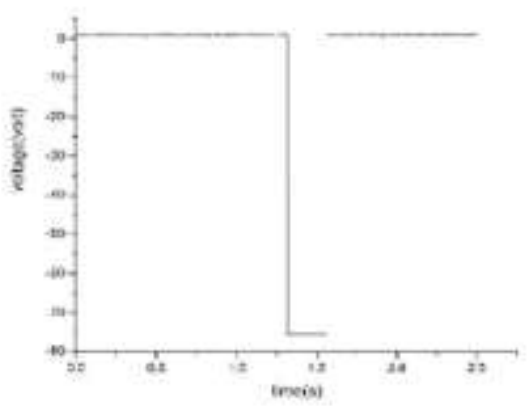

Fig.3 A typical voltage pulse obtained from the pulse forming circuit.

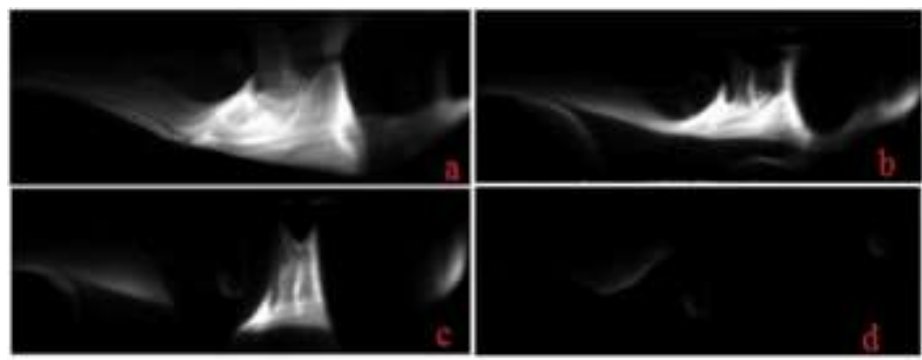

Fig.4. Gradual disappearance of dust layer at discharge voltage of $600 \mathrm{v}$, pulsevoltage 250v and for pulse width of $500 \mathrm{~ms}[\mathrm{t}=0 \mathrm{~s},(\mathrm{a}), \mathrm{t}=0.05 \mathrm{~s}(\mathrm{~b}), \mathrm{t}=0.75 \mathrm{~s}(\mathrm{c}), \mathrm{t}=2 \mathrm{~s}(\mathrm{~d})]$
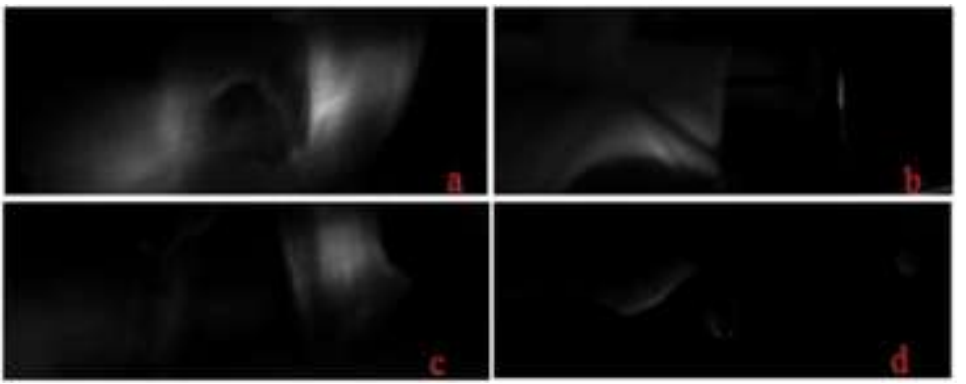

Fig.5. Disappearance of dust for various discharge bias $[750 \mathrm{v}(\mathrm{a}), 700 \mathrm{v}(\mathrm{b}), 650 \mathrm{v}(\mathrm{c}), 600 \mathrm{v}(\mathrm{d})]$ for pulse width at $500 \mathrm{~ms}$ and pulse height is $250 \mathrm{v}$. 
Dust clouds were levitated at four different discharge voltages (at peak-to-peak 750 volt, 700 volt, 650 volt, 600 volt and at pressure $0.2 \mathrm{mbar}$ (flow ratio of argon: acetylene is $7: 2)$ ). At each time single negative pulse (height $50 \mathrm{v}-250 \mathrm{v})$ is given with varying pulse widths $(125 \mathrm{~ms}-5 \mathrm{~s})$ through the electrode for removing dust from a particular region. Initially Stable dust clouds are formed at different discharge voltages at peak-to-peak 750 volt, 700 volt, 650 volt and 600 volt. When pulse is applied particles were start to displace from that particular region depending upon strength of the pulse. It is known that in this type of cogenerated plasma, even larger particles with high relative velocity can agglomerate owing to their coulomb repulsion. So, if sufficient negative pulse height is given to the dust cloud, particles will response to it collectively (they will not repel each other) and displace from plasma confining potential trap. From Fig.4, it can be seen that gradual rejection of dust particles after the application of pulse (here pulse height and width are $250 \mathrm{v}, 500 \mathrm{~ms}$ respectively), at different voltages, from the region where initially a stable dust cloud was present. This reveals that particles can be displaced from a particular region to another. These floating dust particles are completely disappeared at discharge voltage 600v (Fig.4) while at other discharge voltages particles are partially disappeared (Fig.5).The equilibrium of dust cloud in plasma is maintained by various forces (i.e. gravitational, neutral drag, ion drag, thermophoretic, electrostatic etc.) act on the dust particles. We can show that when a positively biased ring electrode is introduced into the dusty plasma cloud it creates a void belowthe

electrode [8] whereas a probe with a negative bias when introduced in plasma it creates a void around it, as shown in Fig.6, and this void is maintained by a balance of the outward Coulomb force on the dust and inward ion drag force due to inward electric field of negatively bias probe [9] $\left(F_{i d}=Q_{D} E\right.$, here, $F_{i d}$ is the ion drag force, $\mathrm{Q}_{\mathrm{D}}$ is the dust charge and $\mathrm{E}$ is the electric field at void edge). A redistribution of charge occur when negative pulse is introduced through a probe inside dusty plasma and thus resulted in a decrement of total electric force (discharge and probe) as well as the ion drag force. This leads to a force inequality in the system and the gravity becomes dominant force which may resulted in disappearance of carbon dust from the region. Again, one cannot draw a direct correlation between the instabilitieindischarge current (which is evident during the experiment) with the dust dynamics during the application of negative pulse [10].

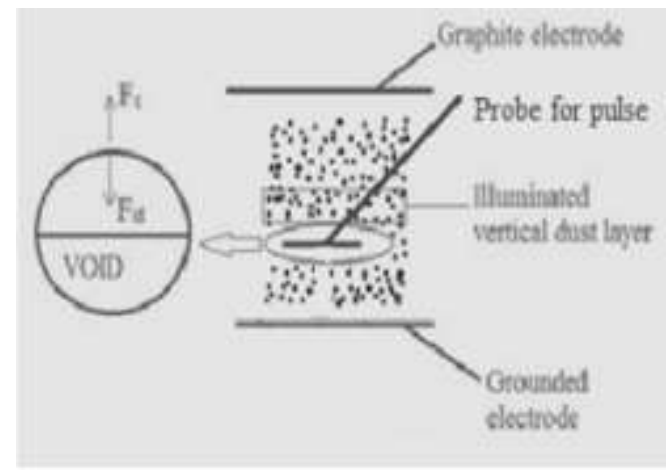

Fig.6: Schematic of physical scenario of typical experimental condition.
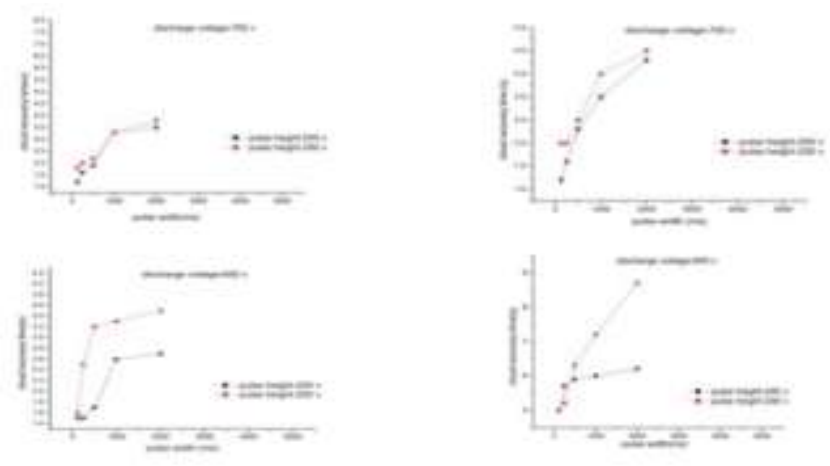

Fig.7. Variation of dust cloud recovery time as a function of pulse width at two different pulse heights and four different discharge voltages. 
A newly generated dust particles fill the space slowly to their initial equilibrium position at the end of each pulse since a continuous sputtering along with dissociation is active always. It is seen from Fig.7 that the cloud recovery time increases with pulse height and width.In this letter, we are to proposing an idea for dust transportation from one region to another region with the help of a negative pulsed bias. This technique may be effective for some laboratory experiments as it gives a mechanism of removing unwanted dust from one place to another.

\section{ACKNOWLEDGEMENTS:}

Authors are extremely thankful to the Board of Research in Fusion Science \& Technology (BRFST), India for the partial financial support provided through their Grant No. NFP Basic-A09-01. Thanks to Dr. Suryakant B. Gupta, Mr. Vishal Jain and Mr. B. K. Patel of FCIPT, Institute for Plasma Research, Gandhinagar for designing the dc pulse circuit.

\section{REFERENCES:}

[1] Y. Kurimoto, N. Matsuda \& G. Uchida et al. (2004) Thin Solid Films 457, 285

[2] Y.-F. Li, U. Konopka \& K. Jiang et al. (2009) Appl. Phys. Lett. 94, 081502.

[3] C. M. Ticos, I. Jepu \& C. P. Lungu et al. (2010) Appl. Phys. Lett. 97, 011501.

[4] R. A. Schill Jr., Laser Part. (2002) Beams 20, 341.

[5] K. B. Chai, W. Choe \& C. R. Seon et al. (2010) Appl. Phys. Lett. 97, 201503.

[6] Sanjib Sarkar, M. Bose \& J. Pramanik et al. (2013) Phys. Plasmas 20, 024506.

[7] Sanjib Sarkar, M. Bose \& S. Mukherjee et al. (2013) Phys. Plasmas 20, 064502.

[8] Sanjib Sarkar, Malay Mondal \& M. Bose et al. (2015) plasma Sources Sci. Technol. 24, 035007

[9] E. Thomas Jr., K. Avinash, \& R. L. Merlino. (2004) Phys. Plasmas 11, 1770.

[10] M. Mikikian, H. Tawidian, \& T. Lecas. (2012) Phys. Rev. Lett. 109, 245007. 